Acta Crystallographica Section E

Structure Reports

Online

ISSN 1600-5368

\section{(E)-4-Methoxy- $N^{\prime}$-[(pyridin-4-yl)methyl- idene]benzohydrazide monohydrate}

\author{
Muhammad Taha, ${ }^{a}$ Humera Naz, ${ }^{a, b}$ Aqilah Abd \\ Rahman, ${ }^{a, b}$ Nor Hadiani Ismail ${ }^{a}$ and Yousuf Sammer ${ }^{\mathrm{c} *}$
}

aAtta-ur-Rahman Research Institute for Natural Products Discovery (RiND), Universiti Tecknologi MARA, Puncak Alam, 42300 Selangor, Malaysia, ${ }^{\mathbf{b}}$ Faculty of Pharmacy, Universiti Tecknologi MARA, Puncak Alam, 42300 Selangor, Malaysia, and ${ }^{\mathrm{C}} \mathrm{H} . \mathrm{E} . J$. Research Institute of Chemistry, International Center for Chemical and Biological Sciences, University of Karachi, Karachi 75270, Pakistan

Correspondence e-mail: dr.sammer.yousuf@gmail.com

Received 6 August 2012; accepted 8 August 2012

Key indicators: single-crystal X-ray study; $T=273 \mathrm{~K}$; mean $\sigma(\mathrm{C}-\mathrm{C})=0.003 \AA$. $R$ factor $=0.047 ; w R$ factor $=0.146 ;$ data-to-parameter ratio $=14.1$.

In the title compound, $\mathrm{C}_{14} \mathrm{H}_{13} \mathrm{~N}_{3} \mathrm{O}_{2} \cdot \mathrm{H}_{2} \mathrm{O}$, the azomethine double bond adopts an $E$ conformation and the $\mathrm{N}-\mathrm{N}=\mathrm{C}-\mathrm{C}$ torsion angle is $178.37(19)^{\circ}$. The dihedral angle between the benzene and pyridine rings is $5.58(12)^{\circ}$ and the $\mathrm{C}$ atom of the methoxy group is roughly coplanar with its attached ring [deviation $=0.157$ (3) $\AA$ ]. In the crystal, the components are linked by $\mathrm{O}-\mathrm{H} \cdots \mathrm{O}, \mathrm{O}-\mathrm{H} \cdots \mathrm{N}, \mathrm{N}-\mathrm{H} \cdots \mathrm{O}$ and $\mathrm{C}-\mathrm{H} \cdots \mathrm{O}$ hydrogen bonds, forming (001) sheets. The water $\mathrm{O}$ atom accepts one $\mathrm{N}-\mathrm{H} \cdots \mathrm{O}$ and two $\mathrm{C}-\mathrm{H} \cdots \mathrm{O}$ interactions from the adjacent organic molecule.

\section{Related literature}

For the biological activity of benzohydraazides, see: Bayrak et al. (2009). For the crystal structures of related benzohydrazides, see: Taha et al. (2012); Fun et al. (2011); Lu et al. (2009); Zhang (2009a,b).<smiles>COc1ccc(C(=O)N/N=C/c2ccncc2)cc1</smiles>

\section{Experimental}

Crystal data

$\mathrm{C}_{14} \mathrm{H}_{13} \mathrm{~N}_{3} \mathrm{O}_{2} \cdot \mathrm{H}_{2} \mathrm{O}$

$M_{r}=273.29$ $a=6.6878(5) \AA$
Monoclinic, $P 2_{1} / c$

$$
\begin{aligned}
& b=7.0420(5) \AA \\
& c=29.249(2) \AA \\
& \beta=94.233(2)^{\circ} \\
& V=1373.74(17) \AA^{3} \\
& Z=4
\end{aligned}
$$

Data collection

Bruker SMART APEX CCD diffractometer

Absorption correction: multi-scan (SADABS; Bruker, 2000)

$T_{\text {min }}=0.981, T_{\max }=0.991$

\section{Refinement}

$R\left[F^{2}>2 \sigma\left(F^{2}\right)\right]=0.047$

$w R\left(F^{2}\right)=0.146$

$S=1.03$

2560 reflections
Mo $K \alpha$ radiation $\mu=0.10 \mathrm{~mm}^{-1}$ $T=273 \mathrm{~K}$ $0.20 \times 0.17 \times 0.10 \mathrm{~mm}$

7767 measured reflections 2560 independent reflections 1548 reflections with $I>2 \sigma(I)$ $R_{\text {int }}=0.038$

\title{
Table 1
}

Hydrogen-bond geometry $\left(\AA{ }^{\circ}\right)$.

\begin{tabular}{lllll}
\hline$D-\mathrm{H} \cdots A$ & $D-\mathrm{H}$ & $\mathrm{H} \cdots A$ & $D \cdots A$ & $D-\mathrm{H} \cdots A$ \\
\hline $\mathrm{O} 1 W-\mathrm{H} 1 \cdots \mathrm{O} 1^{\mathrm{i}}$ & 0.84 & 2.00 & $2.811(2)$ & 162 \\
$\mathrm{O} 1 W-\mathrm{H} 2 \cdots \mathrm{N}{ }^{\text {ii }}$ & 0.91 & 2.11 & $2.956(3)$ & 154 \\
$\mathrm{~N} 1-\mathrm{H} 1 A \cdots \mathrm{O} 1 W$ & 0.86 & 2.08 & $2.911(2)$ & 161 \\
$\mathrm{C} 1-\mathrm{H} 1 B \cdots \mathrm{O} 1 W$ & 0.93 & 2.54 & $3.440(3)$ & 162 \\
$\mathrm{C} 8-\mathrm{H} 8 A \cdots \mathrm{O} 1 W$ & 0.93 & 2.48 & $3.272(3)$ & 143 \\
$\mathrm{C} 11-\mathrm{H} 11 A \cdots \mathrm{O} 2^{\text {iii }}$ & 0.93 & 2.47 & $3.375(3)$ & 165 \\
\hline
\end{tabular}

Symmetry codes: (i) $x+1, y, z$; (ii) $-x+1, y-\frac{1}{2},-z+\frac{1}{2}$; (iii) $x,-y+\frac{1}{2}, z+\frac{1}{2}$.

Data collection: SMART (Bruker, 2000); cell refinement: SAINT (Bruker, 2000); data reduction: $S A I N T$; $\operatorname{program}(\mathrm{s})$ used to solve structure: SHELXS97 (Sheldrick, 2008); program(s) used to refine structure: SHELXL97 (Sheldrick, 2008); molecular graphics: SHELXTL (Sheldrick, 2008); software used to prepare material for publication: SHELXTL, PARST (Nardelli, 1995) and PLATON (Spek, 2009).

Supplementary data and figures for this paper are available from the IUCr electronic archives (Reference: HB6930).

\section{References}

Bayrak, H., Demirbas, N. \& Karaoglu, S. A. (2009). Eur. J. Med. Chem. 44, 4362-4366.

Bruker (2000). SADABS, SMART and SAINT. Bruker AXS Inc., Madison, Wisconsin, USA.

Fun, H.-K., Promdet, P., Chantrapromma, S., Horkaew, J. \& Karalai, C. (2011). Acta Cryst. E67, o3370-03371.

Lu, J.-F., Min, S.-T., Ge, H.-G. \& Ji, X.-H. (2009). Acta Cryst. E65, o2301.

Nardelli, M. (1995). J. Appl. Cryst. 28, 659.

Sheldrick, G. M. (2008). Acta Cryst. A64, 112-122.

Spek, A. L. (2009). Acta Cryst. D65, 148-155.

Taha, M., Naz, H., Abd Rahman, A., Ismaila, N. H. \& Yousuf, S. (2012). Acta Cryst. E68, o2780.

Zhang, X. (2009a). Acta Cryst. E65, o1388.

Zhang, X. (2009b). Acta Cryst. E65, o2200.

$\mathrm{H}$-atom parameters constrained

$\Delta \rho_{\max }=0.16{\mathrm{e} \AA^{-3}}^{-3}$

$\Delta \rho_{\min }=-0.20{\mathrm{e} \AA^{-3}}^{-3}$ 


\title{
supporting information
}

Acta Cryst. (2012). E68, o2778 [doi:10.1107/S1600536812034988]

\section{(E)-4-Methoxy-N'-[(pyridin-4-yl)methylidene]benzohydrazide monohydrate}

\author{
Muhammad Taha, Humera Naz, Aqilah Abd Rahman, Nor Hadiani Ismail and Yousuf Sammer
}

\section{S1. Comment}

The diverse structural features and wide range of biological activities make Benzohydrazides as an importent class of organic compounds. The title compound is an structure analogue of Benzohydrazide, synthesize as a part of our ongoing research to study their varoius biological activities. The structure of title compound (Fig. 1) is similar to that of our recently published benzohydrazide derivative (E)-N'-(3,4-Dimethoxybenzylidene)-4-methoxybenzohydrazide (Taha et al., 2012, Pv2573) with the difference that 3,4-dimethoxy phenyl ring is replaced by pyridine ring (N3/C9-C13). The azomethine (C=N,1.269 (3) $\AA$ ) double bond adopt an $E$ conformation (Fig. 1) with the torsion angle of $178.3(19)^{\circ}(\mathrm{N} 1-$ $\mathrm{N} 2-\mathrm{C} 8-\mathrm{C} 9)$. Phenyl and pyridine rings (C1-C6 and N3/C9-C13) have a dihedral angle of $5.58(12)^{\circ}$ between them and maximum deviation of 0.006 (3) $\AA$ for $\mathrm{C} 13$ atoms from the root mean square plane. The bond lengths and angle were found to be similar as in structurally realted compounds (Fun et al., 2011, Lu et al., 2009, Zhang et al., 2009). In the crystal structure molecules are consolidated by $\mathrm{C} 11-\mathrm{H} 11 \mathrm{~A} \cdots \mathrm{O} 2$ intermolecular hydrogen bonds (Fig.2) and extended to form a two-dimensional-network due to $\mathrm{O} 1 \mathrm{~W}-\mathrm{H} 1 \cdots \mathrm{O} 1$ and $\mathrm{O} 1 \mathrm{~W}-\mathrm{H} 2 \cdots \mathrm{N} 3$ (symmetry codes as in Table 2) intermolecular linkages made by water solvates (Fig. 2).

\section{S2. Experimental}

A mixture of $2 \mathrm{mmol}$ of 4-methoxybenzohydrazide $(0.332 \mathrm{~g}), 2 \mathrm{mmol}$ isonicotinaldehyde $(0.214 \mathrm{~g})$ and catalytical amount of acetic acid was refluxed in methanol $(20 \mathrm{ml})$ for $3 \mathrm{~h}$. The progress of reaction was monitored by TLC. After completion of the reaction, the solvent was evaporated by vacuum to afford the crude product, which was dissolved and recrystallized from methanol to obtain colourless blocks ( $0.418 \mathrm{~g}$ in $82 \%$ yield).

\section{S3. Refinement}

$\mathrm{H}$ atoms on Methyl, phenyl, methine, nitrogen and water were positioned geometrically with $\mathrm{C}-\mathrm{H}=0.95 \AA, \mathrm{CH}_{3}=0.93$ $\AA, \mathrm{NH}=0.86 \AA$ and $\mathrm{O}-\mathrm{H}=0.83-0.90 \AA$ and constrained to ride on their parent atoms with $U_{\text {iso }}(\mathrm{H})=1.5 U_{\text {eq }}\left(\mathrm{CH}_{3}, \mathrm{OH}\right)$ and $1.2 U_{\mathrm{eq}}(\mathrm{CH}, \mathrm{NH})$. A rotating group model was applied to the methyl group. 


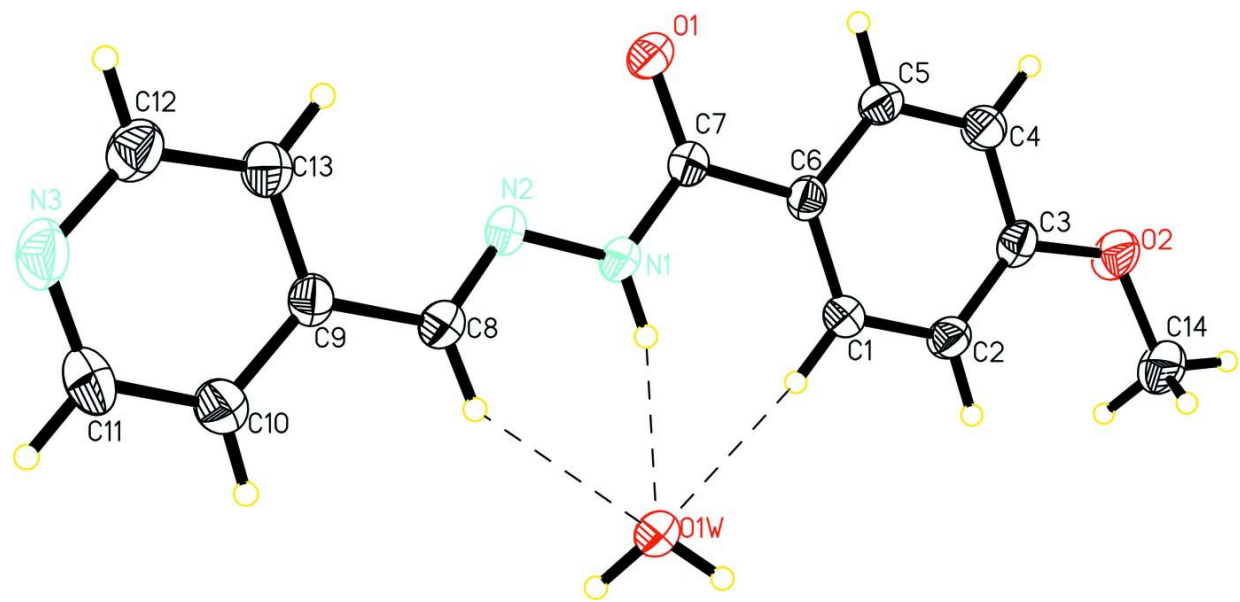

Figure 1

The molecular structure of (I) with displacement ellipsoids drawn at 30\% probability level.

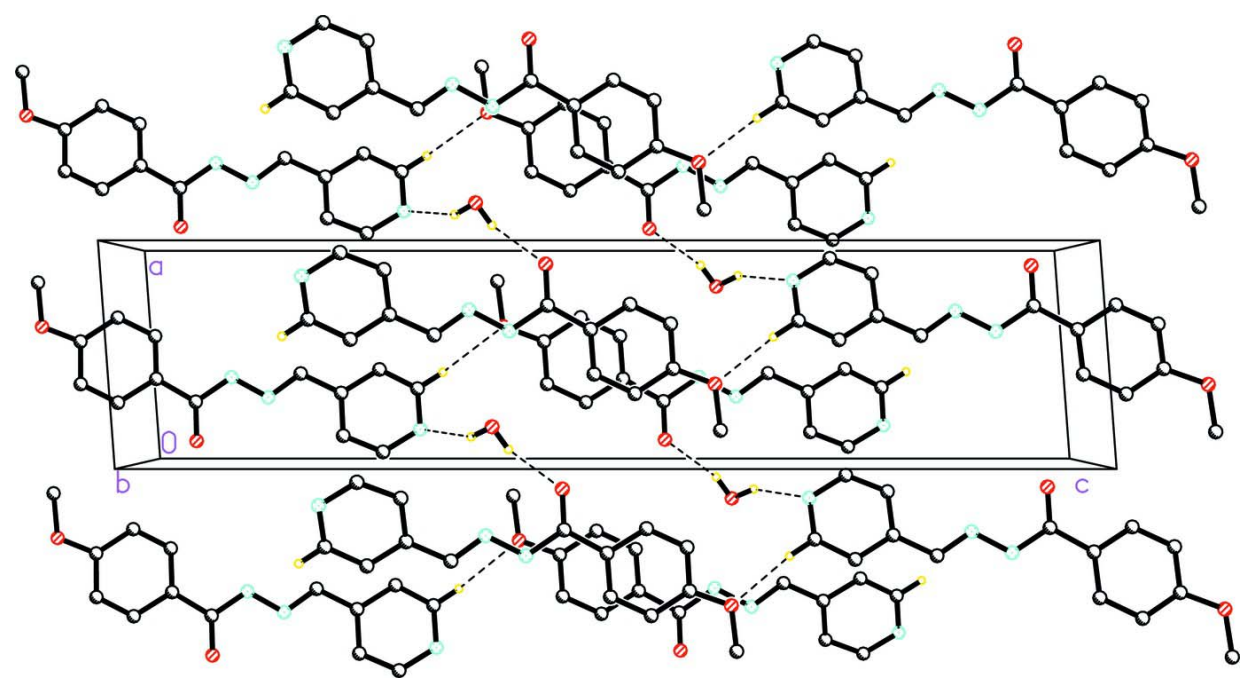

Figure 2

The crystal packing of the title compound I. Only hydrogen atoms involved in hydrogen bonding are shown.

\section{(E)-4-Methoxy- $N^{\prime}-[($ pyridin-4-yl)methylidene]benzohydrazide monohydrate}

Crystal data

$\mathrm{C}_{14} \mathrm{H}_{13} \mathrm{~N}_{3} \mathrm{O}_{2} \cdot \mathrm{H}_{2} \mathrm{O}$

$M_{r}=273.29$

Monoclinic, $P 2_{1} / c$

$a=6.6878(5) \AA$

$b=7.0420(5) \AA$

$c=29.249(2) \AA$

$\beta=94.233(2)^{\circ}$

$V=1373.74(17) \AA^{3}$

$Z=4$
$F(000)=576$

$D_{\mathrm{x}}=1.321 \mathrm{Mg} \mathrm{m}^{-3}$

Mo $K \alpha$ radiation, $\lambda=0.71073 \AA$

Cell parameters from 1112 reflections

$\theta=2.8-22.8^{\circ}$

$\mu=0.10 \mathrm{~mm}^{-1}$

$T=273 \mathrm{~K}$

Block, colourless

$0.20 \times 0.17 \times 0.10 \mathrm{~mm}$ 


\section{Data collection}

Bruker SMART APEX CCD

diffractometer

Radiation source: fine-focus sealed tube

Graphite monochromator

$\omega$ scan

Absorption correction: multi-scan

(SADABS; Bruker, 2000)

$T_{\min }=0.981, T_{\max }=0.991$

\section{Refinement}

Refinement on $F^{2}$

Least-squares matrix: full

$R\left[F^{2}>2 \sigma\left(F^{2}\right)\right]=0.047$

$w R\left(F^{2}\right)=0.146$

$S=1.03$

2560 reflections

182 parameters

0 restraints

Primary atom site location: structure-invariant direct methods
7767 measured reflections

2560 independent reflections

1548 reflections with $I>2 \sigma(I)$

$R_{\text {int }}=0.038$

$\theta_{\max }=25.5^{\circ}, \theta_{\min }=1.4^{\circ}$

$h=-8 \rightarrow 8$

$k=-7 \rightarrow 8$

$l=-35 \rightarrow 35$

Secondary atom site location: difference Fourier map

Hydrogen site location: inferred from neighbouring sites

$\mathrm{H}$-atom parameters constrained

$w=1 /\left[\sigma^{2}\left(F_{0}^{2}\right)+(0.070 P)^{2}\right]$

where $P=\left(F_{\mathrm{o}}^{2}+2 F_{\mathrm{c}}^{2}\right) / 3$

$(\Delta / \sigma)_{\max }<0.001$

$\Delta \rho_{\max }=0.16$ e $\AA^{-3}$

$\Delta \rho_{\min }=-0.20$ e $\AA^{-3}$

Special details

Geometry. All e.s.d.'s (except the e.s.d. in the dihedral angle between two 1.s. planes) are estimated using the full covariance matrix. The cell e.s.d.'s are taken into account individually in the estimation of e.s.d.'s in distances, angles and torsion angles; correlations between e.s.d.'s in cell parameters are only used when they are defined by crystal symmetry. An approximate (isotropic) treatment of cell e.s.d.'s is used for estimating e.s.d.'s involving 1.s. planes.

Refinement. Refinement of $F^{2}$ against ALL reflections. The weighted $R$-factor $w R$ and goodness of fit $S$ are based on $F^{2}$, conventional $R$-factors $R$ are based on $F$, with $F$ set to zero for negative $F^{2}$. The threshold expression of $F^{2}>\sigma\left(F^{2}\right)$ is used only for calculating $R$-factors(gt) etc. and is not relevant to the choice of reflections for refinement. $R$-factors based on $F^{2}$ are statistically about twice as large as those based on $F$, and $R$ - factors based on ALL data will be even larger.

Fractional atomic coordinates and isotropic or equivalent isotropic displacement parameters $\left(\AA^{2}\right)$

\begin{tabular}{lllll}
\hline & $x$ & $y$ & $z$ & $U_{\text {iso }} * / U_{\text {eq }}$ \\
\hline O1 & $0.0974(2)$ & $0.3345(3)$ & $0.05572(5)$ & $0.0607(6)$ \\
O2 & $0.6325(2)$ & $0.1818(3)$ & $-0.10876(5)$ & $0.0590(5)$ \\
$\mathrm{N} 1$ & $0.3945(3)$ & $0.3260(3)$ & $0.09758(6)$ & $0.0446(5)$ \\
$\mathrm{H} 1 \mathrm{~A}$ & 0.5220 & 0.3083 & 0.0985 & $0.053^{*}$ \\
$\mathrm{~N} 2$ & $0.3018(3)$ & $0.3646(3)$ & $0.13646(6)$ & $0.0436(5)$ \\
$\mathrm{N} 3$ & $0.1587(4)$ & $0.5223(4)$ & $0.29858(7)$ & $0.0662(7)$ \\
$\mathrm{C} 1$ & $0.5874(3)$ & $0.2320(3)$ & $0.01504(7)$ & $0.0413(6)$ \\
$\mathrm{H} 1 \mathrm{~B}$ & 0.6640 & 0.2206 & 0.0428 & $0.050^{*}$ \\
$\mathrm{C} 2$ & $0.6754(3)$ & $0.1988(3)$ & $-0.02547(7)$ & $0.0424(6)$ \\
$\mathrm{H} 2 \mathrm{~A}$ & 0.8102 & 0.1659 & -0.0250 & $0.051^{*}$ \\
$\mathrm{C} 3$ & $0.5623(3)$ & $0.2146(3)$ & $-0.06665(7)$ & $0.0424(6)$ \\
$\mathrm{C} 4$ & $0.3619(3)$ & $0.2658(4)$ & $-0.06705(8)$ & $0.0513(7)$ \\
$\mathrm{H} 4 \mathrm{~A}$ & 0.2858 & 0.2773 & -0.0948 & $0.062^{*}$ \\
$\mathrm{C} 5$ & $0.2756(3)$ & $0.2993(3)$ & $-0.02691(8)$ & $0.0462(6)$ \\
$\mathrm{H} 5 \mathrm{~A}$ & 0.1412 & 0.3340 & -0.0277 & $0.055^{*}$ \\
$\mathrm{C} 6$ & $0.3867(3)$ & $0.2821(3)$ & $0.01516(7)$ & $0.0380(5)$
\end{tabular}




$\begin{array}{lllll}\text { C7 } & 0.2800(3) & 0.3160(3) & 0.05702(7) & 0.0411(6) \\ \text { C8 } & 0.4126(4) & 0.3827(4) & 0.17334(7) & 0.0469(6) \\ \text { H8A } & 0.5506 & 0.3663 & 0.1732 & 0.056^{*} \\ \text { C9 } & 0.3230(3) & 0.4292(3) & 0.21603(7) & 0.0429(6) \\ \text { C10 } & 0.4319(4) & 0.4088(4) & 0.25789(8) & 0.0544(7) \\ \text { H10A } & 0.5630 & 0.3639 & 0.2592 & 0.065^{*} \\ \text { C11 } & 0.3438(5) & 0.4556(4) & 0.29754(9) & 0.0650(8) \\ \text { H11A } & 0.4189 & 0.4395 & 0.3253 & 0.078^{*} \\ \text { C12 } & 0.0554(4) & 0.5413(4) & 0.25821(9) & 0.0580(7) \\ \text { H12A } & -0.0747 & 0.5880 & 0.2580 & 0.070^{*} \\ \text { C13 } & 0.1279(4) & 0.4965(4) & 0.21676(8) & 0.0499(6) \\ \text { H13A } & 0.0476 & 0.5110 & 0.1896 & 0.060^{*} \\ \text { C14 } & 0.8422(4) & 0.1550(5) & -0.11117(9) & 0.0640(8) \\ \text { H14A } & 0.8706 & 0.1333 & -0.1424 & 0.096^{*} \\ \text { H14B } & 0.8852 & 0.0473 & -0.0929 & 0.096^{*} \\ \text { H14C } & 0.9124 & 0.2663 & -0.0998 & 0.096^{*} \\ \text { O1W } & 0.8218(2) & 0.2877(3) & 0.12291(5) & 0.0657(6) \\ \text { H1 } & 0.9224 & 0.2994 & 0.1078 & 0.098^{*} \\ \text { H2 } & 0.8700 & 0.2103 & 0.1461 & 0.098^{*}\end{array}$

Atomic displacement parameters $\left(\AA^{2}\right)$

\begin{tabular}{lllllll}
\hline & $U^{11}$ & $U^{22}$ & $U^{33}$ & $U^{12}$ & $U^{13}$ & $U^{23}$ \\
\hline $\mathrm{O} 1$ & $0.0361(10)$ & $0.1027(16)$ & $0.0440(10)$ & $0.0027(10)$ & $0.0085(7)$ & $0.0049(9)$ \\
$\mathrm{O} 2$ & $0.0496(10)$ & $0.0914(15)$ & $0.0373(9)$ & $-0.0042(10)$ & $0.0115(8)$ & $-0.0133(9)$ \\
$\mathrm{N} 1$ & $0.0347(10)$ & $0.0645(15)$ & $0.0357(11)$ & $0.0026(10)$ & $0.0104(8)$ & $0.0015(9)$ \\
$\mathrm{N} 2$ & $0.0415(11)$ & $0.0552(13)$ & $0.0352(11)$ & $0.0018(10)$ & $0.0111(9)$ & $0.0016(9)$ \\
$\mathrm{N} 3$ & $0.0749(17)$ & $0.0805(18)$ & $0.0450(14)$ & $-0.0011(14)$ & $0.0167(12)$ & $-0.0098(12)$ \\
C1 & $0.0384(13)$ & $0.0493(15)$ & $0.0358(12)$ & $0.0007(11)$ & $0.0012(10)$ & $0.0032(10)$ \\
C2 & $0.0360(13)$ & $0.0508(16)$ & $0.0410(13)$ & $0.0012(11)$ & $0.0057(10)$ & $-0.0028(11)$ \\
C3 & $0.0419(13)$ & $0.0485(15)$ & $0.0378(13)$ & $-0.0085(11)$ & $0.0102(10)$ & $-0.0054(11)$ \\
C4 & $0.0428(14)$ & $0.075(2)$ & $0.0359(14)$ & $-0.0049(13)$ & $-0.0009(10)$ & $-0.0017(12)$ \\
C5 & $0.0340(13)$ & $0.0624(18)$ & $0.0424(14)$ & $-0.0034(12)$ & $0.0034(10)$ & $0.0007(12)$ \\
C6 & $0.0378(12)$ & $0.0403(14)$ & $0.0366(12)$ & $-0.0053(11)$ & $0.0068(9)$ & $0.0025(10)$ \\
C7 & $0.0370(13)$ & $0.0488(16)$ & $0.0379(13)$ & $-0.0027(11)$ & $0.0057(10)$ & $0.0049(11)$ \\
C8 & $0.0394(13)$ & $0.0605(17)$ & $0.0416(14)$ & $0.0046(12)$ & $0.0084(11)$ & $0.0005(12)$ \\
C9 & $0.0454(14)$ & $0.0469(16)$ & $0.0370(13)$ & $-0.0016(12)$ & $0.0074(10)$ & $0.0001(11)$ \\
C10 & $0.0537(15)$ & $0.0636(19)$ & $0.0456(15)$ & $0.0042(14)$ & $0.0016(11)$ & $-0.0006(13)$ \\
C11 & $0.081(2)$ & $0.077(2)$ & $0.0368(15)$ & $0.0004(17)$ & $0.0010(13)$ & $-0.0006(13)$ \\
C12 & $0.0553(16)$ & $0.0642(19)$ & $0.0560(17)$ & $-0.0004(14)$ & $0.0137(13)$ & $-0.0108(14)$ \\
C13 & $0.0504(15)$ & $0.0574(17)$ & $0.0423(14)$ & $0.0025(13)$ & $0.0059(11)$ & $-0.0048(12)$ \\
C14 & $0.0565(17)$ & $0.087(2)$ & $0.0516(16)$ & $0.0062(16)$ & $0.0226(13)$ & $-0.0059(14)$ \\
O1W & $0.0371(9)$ & $0.1143(17)$ & $0.0465(10)$ & $0.0079(10)$ & $0.0095(7)$ & $0.0166(10)$ \\
& & & & & &
\end{tabular}

Geometric parameters $(\AA, \stackrel{\circ}{)}$

\begin{tabular}{llll}
\hline $\mathrm{O} 1-\mathrm{C} 7$ & $1.226(2)$ & $\mathrm{C} 5-\mathrm{H} 5 \mathrm{~A}$ & 0.9300 \\
$\mathrm{O} 2-\mathrm{C} 3$ & $1.370(3)$ & $\mathrm{C} 6-\mathrm{C} 7$ & $1.481(3)$
\end{tabular}




\begin{tabular}{|c|c|c|c|}
\hline $\mathrm{O} 2-\mathrm{C} 14$ & $1.422(3)$ & $\mathrm{C} 8-\mathrm{C} 9$ & $1.462(3)$ \\
\hline $\mathrm{N} 1-\mathrm{N} 2$ & $1.362(2)$ & $\mathrm{C} 8-\mathrm{H} 8 \mathrm{~A}$ & 0.9300 \\
\hline $\mathrm{N} 1-\mathrm{C} 7$ & $1.365(3)$ & $\mathrm{C} 9-\mathrm{C} 10$ & $1.385(3)$ \\
\hline $\mathrm{N} 1-\mathrm{H} 1 \mathrm{~A}$ & 0.8600 & $\mathrm{C} 9-\mathrm{C} 13$ & $1.390(3)$ \\
\hline $\mathrm{N} 2-\mathrm{C} 8$ & $1.269(3)$ & $\mathrm{C} 10-\mathrm{C} 11$ & $1.379(3)$ \\
\hline $\mathrm{N} 3-\mathrm{C} 11$ & $1.326(3)$ & $\mathrm{C} 10-\mathrm{H} 10 \mathrm{~A}$ & 0.9300 \\
\hline N3- 12 & $1.330(3)$ & $\mathrm{C} 11-\mathrm{H} 11 \mathrm{~A}$ & 0.9300 \\
\hline $\mathrm{C} 1-\mathrm{C} 2$ & $1.382(3)$ & $\mathrm{C} 12-\mathrm{C} 13$ & $1.375(3)$ \\
\hline $\mathrm{C} 1-\mathrm{C} 6$ & $1.388(3)$ & $\mathrm{C} 12-\mathrm{H} 12 \mathrm{~A}$ & 0.9300 \\
\hline $\mathrm{C} 1-\mathrm{H} 1 \mathrm{~B}$ & 0.9300 & $\mathrm{C} 13-\mathrm{H} 13 \mathrm{~A}$ & 0.9300 \\
\hline $\mathrm{C} 2-\mathrm{C} 3$ & $1.379(3)$ & $\mathrm{C} 14-\mathrm{H} 14 \mathrm{~A}$ & 0.9600 \\
\hline $\mathrm{C} 2-\mathrm{H} 2 \mathrm{~A}$ & 0.9300 & C14-H14B & 0.9600 \\
\hline $\mathrm{C} 3-\mathrm{C} 4$ & $1.387(3)$ & $\mathrm{C} 14-\mathrm{H} 14 \mathrm{C}$ & 0.9600 \\
\hline $\mathrm{C} 4-\mathrm{C} 5$ & $1.367(3)$ & $\mathrm{O} 1 \mathrm{~W}-\mathrm{H} 1$ & 0.8361 \\
\hline $\mathrm{C} 4-\mathrm{H} 4 \mathrm{~A}$ & 0.9300 & $\mathrm{O} 1 \mathrm{~W}-\mathrm{H} 2$ & 0.9098 \\
\hline $\mathrm{C} 5-\mathrm{C} 6$ & $1.395(3)$ & & \\
\hline $\mathrm{C} 3-\mathrm{O} 2-\mathrm{C} 14$ & $118.14(18)$ & $\mathrm{N} 1-\mathrm{C} 7-\mathrm{C} 6$ & $116.9(2)$ \\
\hline $\mathrm{N} 2-\mathrm{N} 1-\mathrm{C} 7$ & $118.33(18)$ & $\mathrm{N} 2-\mathrm{C} 8-\mathrm{C} 9$ & $119.9(2)$ \\
\hline $\mathrm{N} 2-\mathrm{N} 1-\mathrm{H} 1 \mathrm{~A}$ & 120.8 & $\mathrm{~N} 2-\mathrm{C} 8-\mathrm{H} 8 \mathrm{~A}$ & 120.1 \\
\hline $\mathrm{C} 7-\mathrm{N} 1-\mathrm{H} 1 \mathrm{~A}$ & 120.8 & $\mathrm{C} 9-\mathrm{C} 8-\mathrm{H} 8 \mathrm{~A}$ & 120.1 \\
\hline $\mathrm{C} 8-\mathrm{N} 2-\mathrm{N} 1$ & $117.12(19)$ & $\mathrm{C} 10-\mathrm{C} 9-\mathrm{C} 13$ & $117.0(2)$ \\
\hline $\mathrm{C} 11-\mathrm{N} 3-\mathrm{C} 12$ & $116.1(2)$ & $\mathrm{C} 10-\mathrm{C} 9-\mathrm{C} 8$ & $120.7(2)$ \\
\hline $\mathrm{C} 2-\mathrm{C} 1-\mathrm{C} 6$ & $121.2(2)$ & $\mathrm{C} 13-\mathrm{C} 9-\mathrm{C} 8$ & $122.3(2)$ \\
\hline $\mathrm{C} 2-\mathrm{C} 1-\mathrm{H} 1 \mathrm{~B}$ & 119.4 & $\mathrm{C} 11-\mathrm{C} 10-\mathrm{C} 9$ & $119.3(2)$ \\
\hline $\mathrm{C} 6-\mathrm{C} 1-\mathrm{H} 1 \mathrm{~B}$ & 119.4 & $\mathrm{C} 11-\mathrm{C} 10-\mathrm{H} 10 \mathrm{~A}$ & 120.3 \\
\hline $\mathrm{C} 3-\mathrm{C} 2-\mathrm{C} 1$ & $119.6(2)$ & $\mathrm{C} 9-\mathrm{C} 10-\mathrm{H} 10 \mathrm{~A}$ & 120.3 \\
\hline $\mathrm{C} 3-\mathrm{C} 2-\mathrm{H} 2 \mathrm{~A}$ & 120.2 & $\mathrm{~N} 3-\mathrm{C} 11-\mathrm{C} 10$ & $124.1(3)$ \\
\hline $\mathrm{C} 1-\mathrm{C} 2-\mathrm{H} 2 \mathrm{~A}$ & 120.2 & $\mathrm{~N} 3-\mathrm{C} 11-\mathrm{H} 11 \mathrm{~A}$ & 117.9 \\
\hline $\mathrm{O} 2-\mathrm{C} 3-\mathrm{C} 2$ & $124.7(2)$ & $\mathrm{C} 10-\mathrm{C} 11-\mathrm{H} 11 \mathrm{~A}$ & 117.9 \\
\hline $\mathrm{O} 2-\mathrm{C} 3-\mathrm{C} 4$ & $115.6(2)$ & $\mathrm{N} 3-\mathrm{C} 12-\mathrm{C} 13$ & $124.5(3)$ \\
\hline $\mathrm{C} 2-\mathrm{C} 3-\mathrm{C} 4$ & $119.7(2)$ & $\mathrm{N} 3-\mathrm{C} 12-\mathrm{H} 12 \mathrm{~A}$ & 117.8 \\
\hline $\mathrm{C} 5-\mathrm{C} 4-\mathrm{C} 3$ & $120.5(2)$ & $\mathrm{C} 13-\mathrm{C} 12-\mathrm{H} 12 \mathrm{~A}$ & 117.8 \\
\hline $\mathrm{C} 5-\mathrm{C} 4-\mathrm{H} 4 \mathrm{~A}$ & 119.8 & $\mathrm{C} 12-\mathrm{C} 13-\mathrm{C} 9$ & $119.0(2)$ \\
\hline $\mathrm{C} 3-\mathrm{C} 4-\mathrm{H} 4 \mathrm{~A}$ & 119.8 & $\mathrm{C} 12-\mathrm{C} 13-\mathrm{H} 13 \mathrm{~A}$ & 120.5 \\
\hline $\mathrm{C} 4-\mathrm{C} 5-\mathrm{C} 6$ & $120.8(2)$ & $\mathrm{C} 9-\mathrm{C} 13-\mathrm{H} 13 \mathrm{~A}$ & 120.5 \\
\hline $\mathrm{C} 4-\mathrm{C} 5-\mathrm{H} 5 \mathrm{~A}$ & 119.6 & $\mathrm{O} 2-\mathrm{C} 14-\mathrm{H} 14 \mathrm{~A}$ & 109.5 \\
\hline $\mathrm{C} 6-\mathrm{C} 5-\mathrm{H} 5 \mathrm{~A}$ & 119.6 & $\mathrm{O} 2-\mathrm{C} 14-\mathrm{H} 14 \mathrm{~B}$ & 109.5 \\
\hline $\mathrm{C} 1-\mathrm{C} 6-\mathrm{C} 5$ & $118.2(2)$ & $\mathrm{H} 14 \mathrm{~A}-\mathrm{C} 14-\mathrm{H} 14 \mathrm{~B}$ & 109.5 \\
\hline $\mathrm{C} 1-\mathrm{C} 6-\mathrm{C} 7$ & $124.6(2)$ & $\mathrm{O} 2-\mathrm{C} 14-\mathrm{H} 14 \mathrm{C}$ & 109.5 \\
\hline $\mathrm{C} 5-\mathrm{C} 6-\mathrm{C} 7$ & $117.2(2)$ & $\mathrm{H} 14 \mathrm{~A}-\mathrm{C} 14-\mathrm{H} 14 \mathrm{C}$ & 109.5 \\
\hline $\mathrm{O} 1-\mathrm{C} 7-\mathrm{N} 1$ & $121.0(2)$ & $\mathrm{H} 14 \mathrm{~B}-\mathrm{C} 14-\mathrm{H} 14 \mathrm{C}$ & 109.5 \\
\hline $\mathrm{O} 1-\mathrm{C} 7-\mathrm{C} 6$ & $122.1(2)$ & $\mathrm{H} 1-\mathrm{O} 1 \mathrm{~W}-\mathrm{H} 2$ & 101.5 \\
\hline $\mathrm{C} 7-\mathrm{N} 1-\mathrm{N} 2-\mathrm{C} 8$ & $-176.5(2)$ & $\mathrm{C} 1-\mathrm{C} 6-\mathrm{C} 7-\mathrm{O} 1$ & $-169.8(2)$ \\
\hline $\mathrm{C} 6-\mathrm{C} 1-\mathrm{C} 2-\mathrm{C} 3$ & $-0.3(3)$ & $\mathrm{C} 5-\mathrm{C} 6-\mathrm{C} 7-\mathrm{O} 1$ & $9.2(3)$ \\
\hline $\mathrm{C} 14-\mathrm{O} 2-\mathrm{C} 3-\mathrm{C} 2$ & $-9.1(3)$ & $\mathrm{C} 1-\mathrm{C} 6-\mathrm{C} 7-\mathrm{N} 1$ & $10.1(3)$ \\
\hline $\mathrm{C} 14-\mathrm{O} 2-\mathrm{C} 3-\mathrm{C} 4$ & $171.4(2)$ & $\mathrm{C} 5-\mathrm{C} 6-\mathrm{C} 7-\mathrm{N} 1$ & $-170.9(2)$ \\
\hline
\end{tabular}




$\begin{array}{ll}\mathrm{C} 1-\mathrm{C} 2-\mathrm{C} 3-\mathrm{O} 2 & -178.8(2) \\ \mathrm{C} 1-\mathrm{C} 2-\mathrm{C} 3-\mathrm{C} 4 & 0.7(4) \\ \mathrm{O} 2-\mathrm{C} 3-\mathrm{C} 4-\mathrm{C} 5 & 179.1(2) \\ \mathrm{C} 2-\mathrm{C} 3-\mathrm{C} 4-\mathrm{C} 5 & -0.4(4) \\ \mathrm{C} 3-\mathrm{C} 4-\mathrm{C} 5-\mathrm{C} 6 & -0.3(4) \\ \mathrm{C} 2-\mathrm{C} 1-\mathrm{C} 6-\mathrm{C} 5 & -0.4(3) \\ \mathrm{C} 2-\mathrm{C} 1-\mathrm{C} 6-\mathrm{C} 7 & 178.6(2) \\ \mathrm{C} 4-\mathrm{C} 5-\mathrm{C} 6-\mathrm{C} 1 & 0.7(4) \\ \mathrm{C} 4-\mathrm{C} 5-\mathrm{C} 6-\mathrm{C} 7 & -178.4(2) \\ \mathrm{N} 2-\mathrm{N} 1-\mathrm{C} 7-\mathrm{O} 1 & -2.6(3) \\ \mathrm{N} 2-\mathrm{N} 1-\mathrm{C} 7-\mathrm{C} 6 & 177.42(18)\end{array}$

$\mathrm{N} 1-\mathrm{N} 2-\mathrm{C} 8-\mathrm{C} 9$

$\mathrm{N} 2-\mathrm{C} 8-\mathrm{C} 9-\mathrm{C} 10$

$\mathrm{N} 2-\mathrm{C} 8-\mathrm{C} 9-\mathrm{C} 13$

$\mathrm{C} 13-\mathrm{C} 9-\mathrm{C} 10-\mathrm{C} 11$

$\mathrm{C} 8-\mathrm{C} 9-\mathrm{C} 10-\mathrm{C} 11$

$\mathrm{C} 12-\mathrm{N} 3-\mathrm{C} 11-\mathrm{C} 10$

C9- $10-\mathrm{C} 11-\mathrm{N} 3$

$\mathrm{C} 11-\mathrm{N} 3-\mathrm{C} 12-\mathrm{C} 13$

N3-C12-C13-C9

$\mathrm{C} 10-\mathrm{C} 9-\mathrm{C} 13-\mathrm{C} 12$

$\mathrm{C} 8-\mathrm{C} 9-\mathrm{C} 13-\mathrm{C} 12$
$178.37(19)$

$165.7(2)$

$-14.8(4)$

$-0.2(4)$

$179.4(2)$

$0.8(4)$

$-0.7(4)$

$0.1(4)$

$-1.0(4)$

$1.0(4)$

$-178.5(2)$

Hydrogen-bond geometry $(\AA, \stackrel{\circ}{)}$

\begin{tabular}{lllll}
\hline$D-\mathrm{H} \cdots A$ & $D-\mathrm{H}$ & $\mathrm{H} \cdots A$ & $D \cdots A$ & $D-\mathrm{H}^{\cdots} A$ \\
\hline $\mathrm{O} 1 W-\mathrm{H} 1 \cdots \mathrm{O} 1^{\mathrm{i}}$ & 0.84 & 2.00 & $2.811(2)$ & 162 \\
$\mathrm{O} 1 W-\mathrm{H} 2 \cdots \mathrm{N} 3^{\mathrm{ii}}$ & 0.91 & 2.11 & $2.956(3)$ & 154 \\
$\mathrm{~N} 1-\mathrm{H} 1 A \cdots \mathrm{O} 1 W$ & 0.86 & 2.08 & $2.911(2)$ & 161 \\
$\mathrm{C} 1-\mathrm{H} 1 B \cdots \mathrm{O} 1 W$ & 0.93 & 2.54 & $3.440(3)$ & 162 \\
$\mathrm{C} 8-\mathrm{H} 8 A \cdots \mathrm{O} 1 W$ & 0.93 & 2.48 & $3.272(3)$ & 143 \\
$\mathrm{C} 11-\mathrm{H} 11 A \cdots \mathrm{O} 2^{\mathrm{iii}}$ & 0.93 & 2.47 & $3.375(3)$ & 165 \\
\hline
\end{tabular}

Symmetry codes: (i) $x+1, y, z$; (ii) $-x+1, y-1 / 2,-z+1 / 2$; (iii) $x,-y+1 / 2, z+1 / 2$. 